\title{
Improved Supersaturation and Oral Absorption of Dutasteride by Amorphous Solid Dispersions
}

\author{
In-Hwan Beak ${ }^{a}$ and Min-Soo Kim*,b \\ ${ }^{a}$ College of Pharmacy, Chungnam National University; 220 Gung-dong, Yuseong-gu, Daejeon 305-764, Republic of \\ Korea: and ${ }^{b}$ Department of Pharmaceutical Engineering, Inje University; Gimhae, Gyeongnam 621-749, Republic of \\ Korea. $\quad$ Received June 26, 2012; accepted August 14, 2012
}

In this study, amorphous solid dispersions containing dutasteride and various excipients, manufactured by spray-drying processes, were characterized to determine the effects on their ability to form supersaturated solutions and to identify the effects of supersaturation on increasing the bioavailability of dutasteride. The excipients included Eudragit E, hydroxypropyl- $\beta$-cyclodextrin (HP- $\beta$-CD), hydroxypropyl cellulose (HPC), hydroxypropylmethyl cellulose (HPMC), and polyvinylpyrrolidone (PVP K30). A solid dispersion with Eudragit $\mathbf{E}$ displayed a high maximum supersaturation with extended supersaturation, compared with a water-soluble polymer. The maximum concentration and the degree of supersaturation increased in the following order: PVP K30 $<\mathrm{HP}-\boldsymbol{\beta}-\mathrm{CD}=\mathrm{HPC}<\mathrm{HPMC}<$ Eudragit E. Oral drug absorption of solid dispersions was obviously higher when compared with micronized raw material and physical mixtures, in the following order: HPC $<$ HPMC $<$ Eudragit E. In fact, the $A U C_{0 \rightarrow 24 \mathrm{~h}}$ and $C_{\max }$ of dutasteride increased with supersaturation concentration. These results suggest that amorphous solid dispersions containing Eudragit $E$, formed by a spray-drying process, offer enhanced supersaturation characteristics, leading to increased oral absorption of dutasteride.

Key words dutasteride; supersaturation; amorphous; poorly water-soluble drug; solid dispersion

Dutasteride (17b- $N$-(2,5-bis-(trifluoromethyl)phenylcarbamoyl)-4-aza-5-and-rost-1-en-3-one) is a synthetic 4-azasteroid compound that inhibits steroid $5-\alpha$ reductase $(5-\mathrm{AR}){ }^{1)}$ It is used for the treatment of benign prostatic hyperplasia (BPH) and hair loss, by inhibiting 5-AR. ${ }^{2)}$ Although dutasteride has many advantages, it is presently marketed only as a soft gelatin capsule that contains $0.5 \mathrm{mg}$ dutasteride in a mixture of oils, such as mono-diglycerides of caprylic/capric acid and butylated hydroxytoluene, because it is insoluble in water (solubility below the quantitation limit of the assay, $0.038 \mathrm{ng} / \mathrm{mL}$ ). ${ }^{3)}$ A capsule filled with a solution usually has limited stability and a relatively short shelf-life period. Soft gelatin capsules also have problems with cross-linking of gelatin, resulting in decreased solubility of gelatin and a reduced release rate of the drug. ${ }^{4)}$

To deliver dutasteride orally in a solid dosage form, the major problems of the dissolution and absorption of dutasteride must be overcome. Many technological methods have been reported with regard to enhancing the dissolution and absorption properties of drugs with low water solubility, such as self-emulsifying formulations, solid dispersions, ionic inclusion complexation, lipid-based complexation, formation of salts, polymorphs, cocrystals, and prodrugs, and particle size reduction techniques, such as micronization or nanomilling. ${ }^{5)}$ Among these, solid dispersions with hydrophilic carriers are particularly promising methods for improving the solubility and dissolution rate of poorly water-soluble drugs. ${ }^{6,7)}$ Solid dispersion formulations currently on the market include griseofulvin in polyethylene glycol (PEG) (Gris-PEG, Novartis), nabilone in povidone (Cesamet, Eli Lilly), and itraconzaole in PEG and hydroxypropylmethyl celllulose (HPMC) (Sporanox, Janssen). ${ }^{8)}$

The objectives of this study were to investigate the effects of various hydrophilic carriers on the maximum degree and

The authors declare no conflict of interest. extent of supersaturation of dutasteride and to develop an effective solid dispersion formulation improving the dissolution and oral absorption of dutasteride. Solid dispersions with various hydrophilic excipients were prepared using spray drying and their supersaturated dissolution profiles were evaluated in comparison with micronized raw material and physical mixtures. In vitro and in vivo correlations were also examined in rats.

\section{Experimental}

Materials Dutasteride was obtained from Dr. Reddy's Laboratories Ltd. (purity 99.6\%, India). Eudragit E PO (Röhm $\mathrm{GmbH}$ and Co., KG, Sontheim, Germany), hydroxypropyl- $\beta$ cyclodextrin with a molar degree of substitution (MS, defined as number of substituent per glucose unit) of 0.65 (HP- $\beta-\mathrm{CD}$, Kleptose $^{\circledR}$ HPB, Roquette, Lestrem, France), hydroxypropyl cellulose (NISSO HPC-SL, Nippon Soda Co., Japan), hydroxypropylmethyl cellulose (HPMC 2910, Shin-Etsu Chemical Co., Japan) and polyvinylpyrrolidone (PVP K30, BASF Co., Ltd., Germany) were used. Finasteride (internal standard (IS)) were obtained from Sigma Chemical Co. (St. Louis, MO, U.S.A.) All organic solvents were HPLC grade. All other chemicals were analytical grade, and double-distilled water was used throughout the study.

Preparation of Solid Dispersions Table 1 lists the solid dispersions investigated in this study. The compositions were prepared by dissolving dutasteride and excipients at a $1: 10 \mathrm{API} /$ excipient ratio and $2.0 \%$ solids in ethanol or a $50 / 50$ mixture of ethanol/dichloromethane. Spray drying was carried out using a laboratory-scale spray dryer (SD 1000, Eyela, Japan) under the following conditions: drug solution concentration, $100 \mathrm{mg} / \mathrm{mL}$; inlet temperature, $70-75^{\circ} \mathrm{C}$; outlet temperature, $55-65^{\circ} \mathrm{C}$; feed rate, $2-3 \mathrm{~mL} / \mathrm{min}$; atomization air pressure, $10 \mathrm{kPa}$; and drying air flow rate, $0.60-0.70 \mathrm{~m}^{3} / \mathrm{min}$. Physical mixtures were also prepared by mixing dutasteride with excipients in the same ratio with a mortar and pestle 
until homogeneous mixtures were obtained. All samples were passed through a $250-\mu \mathrm{m}$ sieve (mesh size 60 ) and then stored in a desiccator at room temperature.

Drug Content The drug content in the solid dispersions was determined by HPLC. A sample of approximately $20 \mathrm{mg}$ was dissolved in $100 \mathrm{~mL}$ of ethanol or ethanol-dichloromethane $(1: 1)$. HPLC analyses of in vitro samples of dutasteride were performed using a HPLC system consisting of a LC10ADvp pump, SIL-10A autoinjector, and SPD-10ADvp UV detector (Shimadzu, Japan). A $\mathrm{C}_{18}$ analytical column (Inertsil ODS-3, $5 \mu \mathrm{m}, 4.6 \times 150 \mathrm{~mm}$, GL Sciences Inc., Japan) was used at room temperature. The mobile phase was $60 \%$ acetonitrile and $40 \%$ water. The injection volume was $20 \mu \mathrm{L}$ or $50 \mu \mathrm{L}$, and the eluent flow rate was $1.0 \mathrm{~mL} / \mathrm{min}$. The signal was monitored at $210 \mathrm{~nm}$.

Particle Size Measurement The particle size of micronized dutasteride and spray-dried solid dispersion particles was determined using a HELOS laser diffraction analyzer (Sympatec $\mathrm{GmbH}$, Germany) equipped with a RODOS vibrating trough disperser. An air pressure of $0.1 \mathrm{MPa}$ and a vacuum of $5.3 \mathrm{kPa}$ were used to ensure that each sample had a uniform powder dispersion.

Surface Area Analysis The specific surface area of samples was determined by $\mathrm{N}_{2}$ adsorption using Surface Area Analyzer ASAP 2010 (Micromeritics Instrument Corporation, U.S.A.)

Powder X-Ray Diffraction (PXRD) X-Ray diffraction patterns were recorded on a Rigaku Powder X-ray diffraction system (Model D/MAX-2200 Ultima/PC, Japan) with Nifiltered $\mathrm{Cu} K \alpha$ radiation. The $2 \theta$ scan range was $5-50^{\circ}$ with a step size of $0.02^{\circ}$.

Supersaturated Dissolution Test Supersaturated dissolution tests were performed using a USP XXIII rotating paddle apparatus with a VK 7000 dissolution testing station and VK $750 \mathrm{~d}$ heater/circulator (Vankel, U.S.A.) at $37^{\circ} \mathrm{C}$ and a rotating speed of $50 \mathrm{rpm}$ in $300 \mathrm{~mL}$ of $\mathrm{pH} 1.2$ simulated gastric fluids (without pepsin). Accurately weighed samples containing the equivalent of $30 \mathrm{mg}$ dutasteride were dispersed in the dissolution medium. Then, 3-mL aliquot samples were collected at different time intervals and filtered using $0.22-\mu \mathrm{m}$ nylon syringe filter. The filtrate was diluted with methanol, and the dutasteride was quantified using HPLC.

In Vivo Animal Studies Male Sprague-Dawley rats weighing 240-260g were obtained from Samtaco Bio Korea Inc. (Korea). All animal experiments were performed according to the "Guidelines for the Care and Use of Laboratory Animals" at Chungnam National University. The rats were deprived of food for $24 \mathrm{~h}$ before the experiment, and food was re-offered at $4 \mathrm{~h}$ post-dosing. The rats were divided into five groups of five animals each. After anesthesia with diethylether, the femoral artery was cannulated with a 23gauge polyethylene cannula. The cannula was flushed with $0.3 \mathrm{~mL}$ of heparin (50 IU) saline solution to prevent blood clotting. After rats recovered from the anesthesia, gelatin minicapsules (Size 9, Torpac, Fairfield, NJ, U.S.A.) filled with micronized raw material, physical mixture or solid dispersions equivalent to $2 \mathrm{mg} / \mathrm{kg}$ of dutasteride were administered orally to rats using a minicapsule dosing syringe. After administration of the capsules, the rats were immediately given $1 \mathrm{~mL}$ of distilled water. Serial blood samples (approximately $400 \mu \mathrm{L}$ each) were collected from the femoral artery before dosing and at $0.5,1,2,3,4,5,12$, and $24 \mathrm{~h}$ post-dosing. The blood samples were placed in heparinized tubes, and the plasma was separated by centrifugation (12000 rpm, $2 \mathrm{~min}$ ). Plasma samples were stored at $-70^{\circ} \mathrm{C}$ until analysis.

LC-MS/MS Analysis The dutasteride concentrations in plasma samples were determined by LC/MS/MS as reported previously, with slight modifications. ${ }^{9)}$ In a microcentrifuge tube, $100-\mu \mathrm{L}$ plasma samples were mixed with $20 \mu \mathrm{L}$ of IS ( $200 \mathrm{ng} / \mathrm{mL}$ finasteride in $50 \%$ methanol), and the tube was vortexed for $30 \mathrm{~s}$. For liquid/liquid extraction of dutasteride and the IS, $100 \mu \mathrm{L}$ of $1 \mathrm{M} \mathrm{NaOH}$ and $600 \mu \mathrm{L}$ of a mixture of methyl tert-butyl ether and methylene chloride $(7: 3)$ were added and mixed using a rotator for $10 \mathrm{~min}$. After centrifugation $(13000 \mathrm{rpm}, 5 \mathrm{~min})$, the supernatant was transferred and evaporated to dryness under a nitrogen stream at $40{ }^{\circ} \mathrm{C}$. The dried extract was resuspended in $200 \mu \mathrm{L}$ of $50 \%$ methanol, and then $10 \mu \mathrm{L}$ was analyzed by LC-MS/MS. An 1100-series liquid chromatography system (Agilent Technologies, Santa Clara, CA, U.S.A.), consisting of a solvent degasser, binary pumps, an autosampler, a column heater, and Phenomenex Kinetex $\mathrm{C}_{18}$ column $(50 \times 2.1 \mathrm{~mm}, 2.6 \mu \mathrm{m})$ was used for the chromatographic separation of dutasteride and the IS (finasteride). The isocratic mobile phase contained $0.1 \%$ formic acid in a mixture of acetonitrile and purified water $(70: 30, \mathrm{v} / \mathrm{v})$ at a flow rate of $0.3 \mathrm{~mL} / \mathrm{min}$. The column and autosampler tray temperatures were maintained at $25^{\circ} \mathrm{C}$ and $4^{\circ} \mathrm{C}$, respectively. The plasma concentrations of dutasteride were quantified using liquid chromatography-mass spectrometry using a PE SCIEX API 2000 (triple-quadrupole) LC-MS/MS system (Applied Biosystems, Foster City, CA, U.S.A.) equipped with an electrospray ionization interface to generate positive ions $[\mathrm{M}+\mathrm{H}]^{+}$. The turbo-ion spray interface was operated in the positive ion mode at $5500 \mathrm{~V}$ and $350^{\circ} \mathrm{C}$. The operating conditions, optimized with a flow injection of a mixture of all analytes, were as follows: nebulizing, auxiliary, and curtain gas flows of 1.04, 4.0, and $10.0 \mathrm{~L} / \mathrm{min}$, respectively; collision gas (nitrogen) pressure, $6 \times 10^{-5}$ Torr; orifice voltage (declustering potential), $111 \mathrm{~V}$; ring voltage (focusing potential), $330 \mathrm{~V}$; entrance potential, $11 \mathrm{~V}$; collision energy, $33 \mathrm{~V}$; and collision exit

Table 1. Drug Content, Particle Size, and Specific Surface Area of Micronized Drug and Solid Dispersions Prepared by Spray Drying

\begin{tabular}{lccc}
\hline \hline Composition $(1: 10, \mathrm{w} / \mathrm{w})$ & Drug content $^{a)}$ & Volume mean particle size $\left.^{a}\right)(\mu \mathrm{m})$ & ${\left.\text { Specific surface } \operatorname{area}^{a}\right)\left(\mathrm{m}^{2} / \mathrm{g}\right)}$ \\
\hline Drug: Eudragit E & $98.5 \pm 0.8 \%$ & $4.84 \pm 0.91(2.01)^{b)}$ & $2.41 \pm 0.03$ \\
Drug: HP- $\beta$-CD & $97.6 \pm 1.2 \%$ & $5.21 \pm 0.59(2.51)$ & $3.75 \pm 0.09$ \\
Drug: HPC & $99.2 \pm 0.5 \%$ & $4.56 \pm 0.88(1.98)$ & $3.38 \pm 0.06$ \\
Drug: HPMC & $98.8 \pm 1.1 \%$ & $6.37 \pm 1.09(2.33)$ & $2.32 \pm 0.03$ \\
Drug: PVP K30 & $98.1 \pm 1.0 \%$ & $3.65 \pm 0.93(2.31)$ & $2.52 \pm 0.03$ \\
Micronized raw drug & - & $3.32 \pm 0.29(2.04)$ & $3.81 \pm 0.07$ \\
\hline
\end{tabular}

a) Mean \pm S.D. $n=3 . b)$ SPAN $=\left(d_{90}-d_{10}\right) / d_{50}$, where $d_{10}, d_{50}$ and $d_{90}$ are the diameter sizes and the given percentage value is the percentage of particles smaller than that size. 
potential, 5.0 V. Quantitation was performed by multiple reaction monitoring (MRM) of the protonated precursor. The mass transitions used for dutasteride and the internal standard were $m / z 529.3 \rightarrow 461.3$ and $373.2 \rightarrow 305.3$, respectively (dwell time, $150 \mathrm{~ms}$ ). Quadrupoles Q1 and Q3 were set at unit resolution. The analytical data were processed using the Analyst 1.4.1 software (ABI, Inc.). Dutasteride plasma concentrations were quantified by comparison with standard curves generated from spiked plasma samples extracted in the same manner as the test samples. A calibration curve $(1-500 \mathrm{ng} / \mathrm{mL})$ was generated from the standard solutions to confirm a linear relationship between the peak area ratio and the concentration of dutasteride. The slope, intercept, and coefficient of determination $\left(r^{2}\right)$ were calculated as regression parameters of a weighted $(1 / y)$ linear regression $\left(r^{2}>0.99\right)$.

Data Analysis The $A U C_{0 \rightarrow 24 \mathrm{~h}}$ was calculated using noncompartmental analysis (WinNonlin 2.1; Pharsight Corp., Mountain View, CA, U.S.A.). The $C_{\max }$ and $T_{\max }$ were obtained directly from blood data. To demonstrate statistically significant differences, one-way analysis of variance (ANOVA) followed by LSD test and the Student-Newman-Keul test was performed, using SPSS 12.0 software (SPSS, Chicago, IL, U.S.A.).

\section{Results and Discussion}

A spray-drying process was used to manufacture the compositions of dutasteride listed in Table 1. HPLC analysis was used to determine the exact content of dutasteride in the dispersions, and revealed that the content was in good agreement with the theoretical values. The mean values of particle size and the specific surface area of solid dispersions prepared using spray drying were $3.65-6.37 \mu \mathrm{m}$ and $2.32-3.38 \mathrm{~m}^{2} / \mathrm{g}$, respectively. Figure 1 shows the PXRD patterns of the raw material and of solid dispersions prepared by the spray-drying process. The diffraction pattern of crystalline dutasteride showed characteristic high-intensity peaks at $13.42^{\circ}, 16.02^{\circ}$, $17.10^{\circ}, 19.66^{\circ}$, and $23.56^{\circ}$ of $2 \theta$. In contrast, the PXRD patterns of solid dispersions were completely different from those of dutasteride and exhibited no characteristic diffraction peak corresponding to crystalline dutasteride. These data confirm that dutasteride was present in an amorphous form within the solid dispersions.

Supersaturated dissolution studies were conducted in acidic media for $24 \mathrm{~h}$ for each of the solid dispersions prepared using spray drying, as well as the physical mixture (Fig. 2, Table 2). The degree of supersaturation is defined as the concentration of dutasteride in the dissolution media divided by the equilibrium solubility of dutasteride within the medium. In this study, the equilibrium solubility of the physical mixture was used instead that of crystalline dutasteride because the concentration of micronized dutasteride was not detected in the dissolution media. All solid dispersions had rapid dissolution rates, reaching maximum supersaturation within $1 \mathrm{~h}$. The ANOVA test revealed significant differences among the samples $(p<0.001)$, and the maximum concentration and the degree of supersaturation increased in the following order: PVP $\mathrm{K} 30<\mathrm{HP}-\beta-\mathrm{CD}=\mathrm{HPC}<\mathrm{HPMC}<$ Eudragit $\mathrm{E}$ (ranked by the Student-Newman-Keuls test). Eudragit E solid dispersions displayed a high maximum supersaturation with an extended supersaturation that produced the highest area under the curve $(A U C)$ of supersaturated dissolution of all the compositions

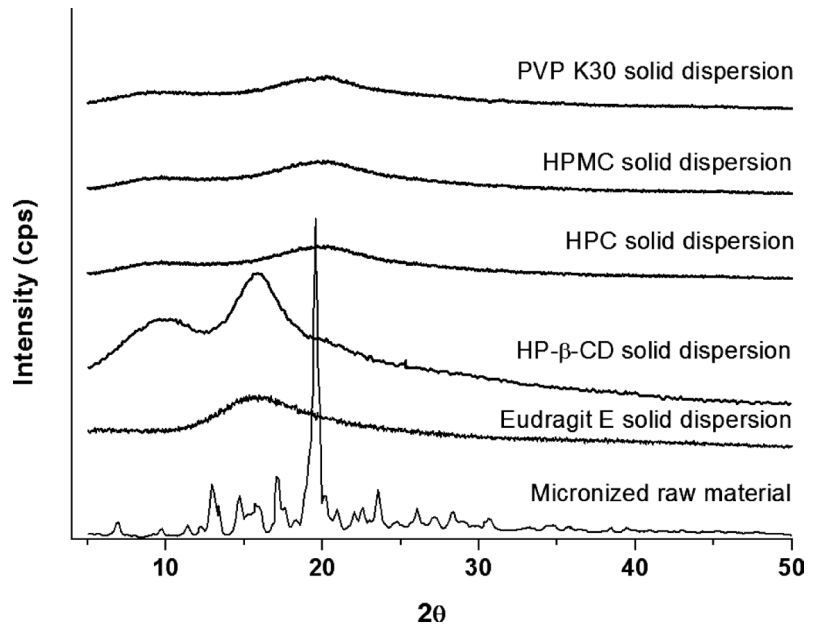

Fig. 1. Powder X-Ray Diffraction Patterns of Micronized Raw Material and Solid Dispersions Prepared Using a Spray-Drying Process

\section{investigated.}

Generally, a supersaturated drug solution is thermodynamically unstable and has a tendency to return to the equilibrium state, with drug precipitation. ${ }^{10)}$ During drug precipitation, polymers may adsorb onto the surface of small embryo particles and inhibit crystal growth by blocking the active surface and providing steric stabilization. ${ }^{11)}$ In addition, hydrogen bonding between the drug and polymers may compliment hydrophobic interactions between these species, helping to maintain supersaturation. Among the polymers used, Eudragit $\mathrm{E}$ is a cationic copolymer based on dimethylaminoethyl methacrylate, butyl methacrylate, and methyl methacrylate. Structurally, Eudragit E has an aminoalkyl group, which is a proton-accepting group for dutasteride. Dutasteride has a 2,5-bis-(trifluoromethyl)phenyl group, which is a protondonating group. ${ }^{12)}$ It has been suggested that intermolecular interactions could occur between dutasteride and Eudragit E, such as electrostatic interactions or hydrogen bonding, in addition to a hydrophobic interaction, which will provide a high maximum supersaturation with an extended supersaturation. ${ }^{10)}$ However, further study on the formation and stabilization mechanism of the Eudragit E solid dispersion is needed.

Next, the bioavailability of dutasteride solid dispersions was evaluated. Figure 3 presents the plasma concentrationtime profile of dutasteride in rats after oral administration of micronized raw material, physical mixture, and solid dispersions at a dose equivalent to $2 \mathrm{mg} / \mathrm{kg}$ dutasteride. Table 3 lists the pharmacokinetic parameters calculated from the time profiles. The oral drug absorption of solid dispersions was obviously higher than with micronized raw material or the physical mixture. For the micronized raw material, the $A U C_{0 \rightarrow 24 \mathrm{~h}}, C_{\max }$, and $T_{\max }$ were $292.1 \pm 70.4 \mathrm{ng} \cdot \mathrm{h} / \mathrm{mL}, 14.8 \pm$ $4.4 \mathrm{ng} / \mathrm{mL}$, and $10.0 \pm 2.7 \mathrm{~h}$, respectively. After administration of the HPMC solid dispersion, the $A U C_{0 \rightarrow 12 \mathrm{~h}}, C_{\max }$, and $T_{\max }$ were $1775.0 \pm 508.7 \mathrm{ng} \cdot \mathrm{h} / \mathrm{mL}, 104.2 \pm 6.0 \mathrm{ng} / \mathrm{mL}$, and $9.0 \pm$ 2.7 h, respectively. Furthermore, the Eudragit E solid dispersion exhibited higher bioavailability than the raw material, with approximately 8.0- and 8.1-fold increases in $A U C_{0 \rightarrow 12} \mathrm{~h}$ and $C_{\max }$. Based on ANOVA, significant differences appeared among the samples $(p<0.05)$, which were ranked by the Student-Newman-Keul test in order of increasing $A U C_{0 \rightarrow 24 \mathrm{~h}}$ as follows: micronized raw material $=$ physical mixture $<\mathrm{HPC}$ 
(a)

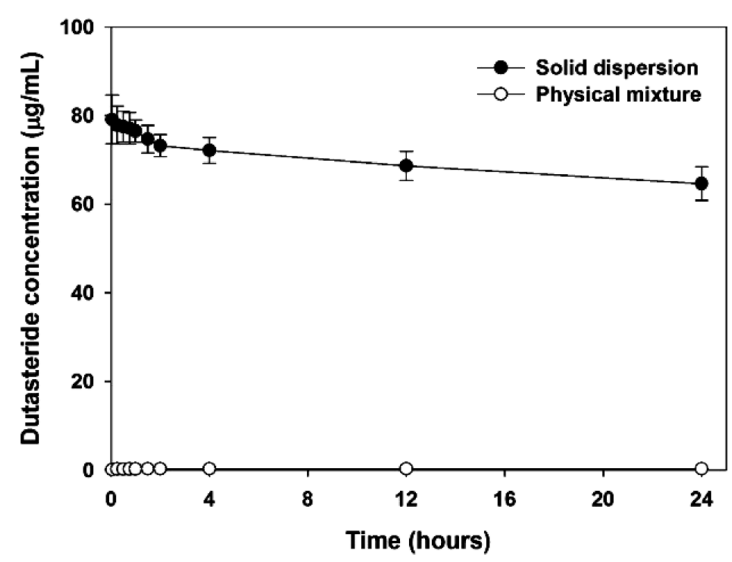

(c)

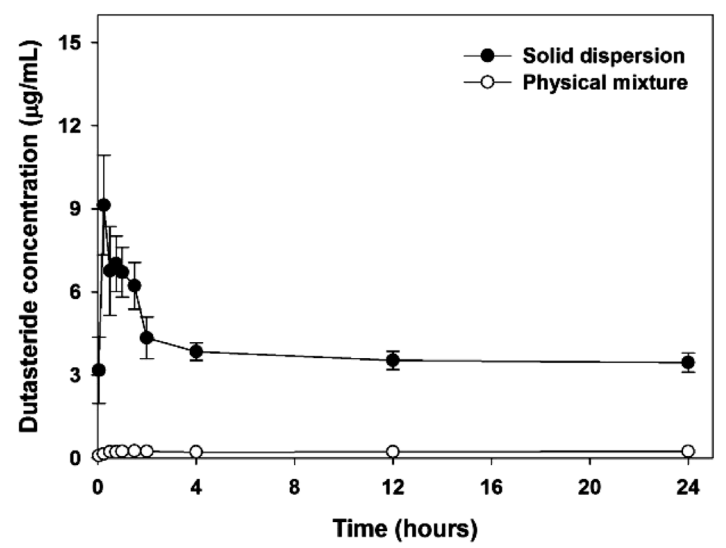

(e)

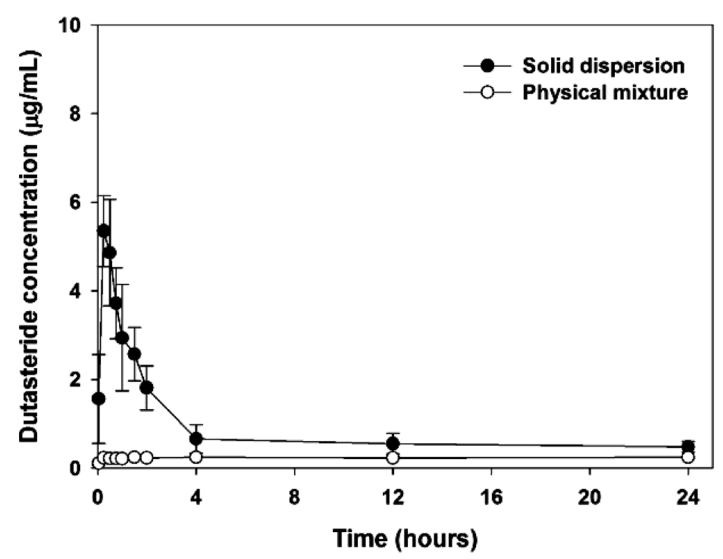

(b)

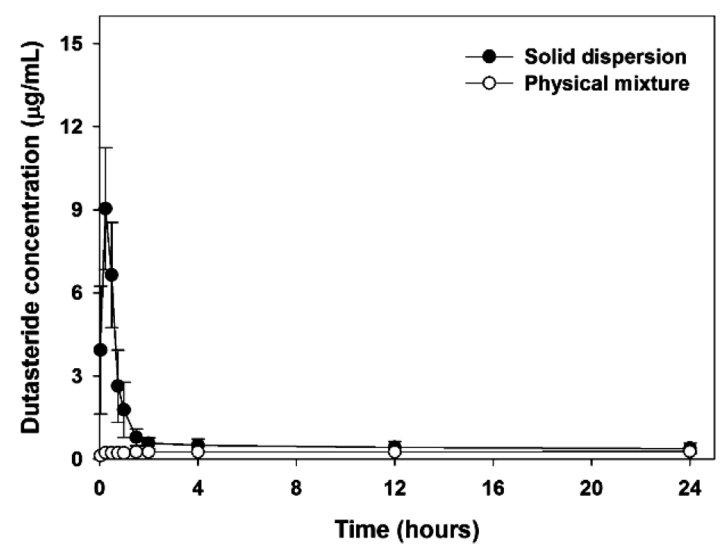

(d)

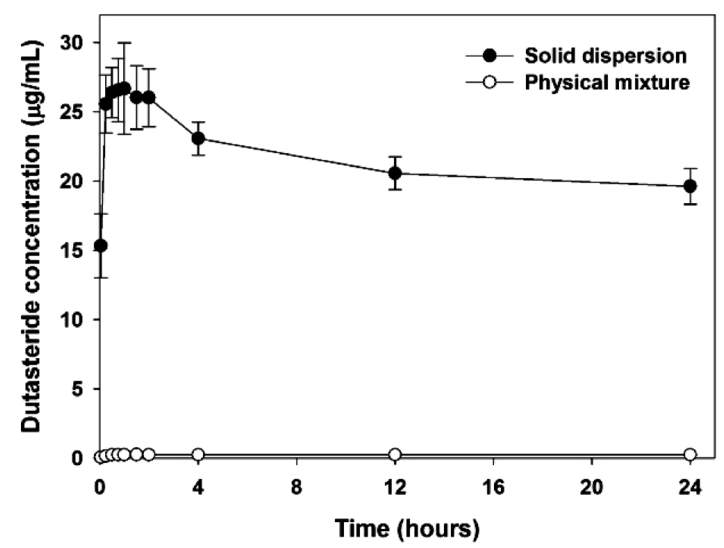

Fig. 2. Supersaturated Dissolution of Physical Mixture and Solid Dispersions; (a) Eudragit E, (b) HP- $\beta$-CD, (c) HPC, (d) HPMC, and (e) PVP K30 (Data Represented Mean \pm S.D., $n=3$ )

solid dispersion $<$ HPMC solid dispersion $<$ Eudragit $E$ solid dispersion. In fact, the $A U C_{0 \rightarrow 24 \mathrm{~h}}$ and $C_{\max }$ of dutasteride increased with supersaturation concentration. The bioavailability of Eudragit E solid dispersion was markedly higher than that of HPMC and HPC solid dispersion, because of the higher supersaturation concentration over a longer time. Generally, the formation of a supersaturated state with solid dispersions may increase oral bioavailability of active pharmaceutical ingredients that suffer from poor bioavailability. ${ }^{11,13,14)}$ The Eudragit E solid dispersion with amorphous dutasteride formed 
Table 2. Supersaturated Dissolution Data of Solid Dispersion and Physical Mixture

\begin{tabular}{|c|c|c|c|}
\hline \multirow{2}{*}{ Composition $(1: 10, w / w)$} & \multicolumn{2}{|c|}{ Solid dispersion } & \multirow{2}{*}{$\begin{array}{c}\text { Physical mixture } \\
\text { Equilibrium solubility }^{a}(\mu \mathrm{g} / \mathrm{mL})\end{array}$} \\
\hline & Maximum concentration $\left.^{a}\right)(\mu \mathrm{g} / \mathrm{mL})$ & The degree of supersaturation ${ }^{b)}$ & \\
\hline Drug: Eudragit E & $79.10 \pm 5.5$ & 304.2 & $0.26 \pm 0.03$ \\
\hline Drug:HP-B-CD & $9.04 \pm 2.2$ & 33.5 & $0.27 \pm 0.04$ \\
\hline Drug: HPC & $9.13 \pm 1.8$ & 36.5 & $0.25 \pm 0.05$ \\
\hline Drug: HPMC & $26.68 \pm 3.3$ & 111.2 & $0.24 \pm 0.02$ \\
\hline Drug:PVP K30 & $5.25 \pm 0.8$ & 22.8 & $0.23 \pm 0.04$ \\
\hline
\end{tabular}

a) Mean \pm S.D. $n=3 . b$ ) The degree of supersaturation is defined as the maximum concentration of dutasteride (solid dispersion) in the dissolution media divided by the equilibrium solubility of dutasteride (physical mixture) within medium.

Table 3. Pharmacokinetic Parameters of Dutasteride in Rats after Oral Administration of Micronized Drug, Physical Mixture, or Solid Dispersions

\begin{tabular}{lccr}
\hline \hline \multirow{2}{*}{ Formulation } & \multicolumn{3}{c}{ Pharmacokinetic parameter ${ }^{a)}$} \\
\cline { 2 - 4 } & $C_{\max }(\mathrm{ng} / \mathrm{mL})$ & $A U C_{0 \rightarrow 24 \mathrm{~h}}(\mathrm{ng} \cdot \mathrm{h} / \mathrm{mL})$ & $T_{\max }(\mathrm{h})$ \\
\hline Drug : Eudragit E solid dispersion & $118.8 \pm 8.8^{b-e)}$ & $2331.8 \pm 227.9^{b-e)}$ & $10.0 \pm 2.7$ \\
Drug: HPMC solid dispersion & $104.2 \pm 6.0^{b-d)}$ & $1775.0 \pm 508.7^{b-d)}$ & $9.0 \pm 2.7$ \\
Drug: HPC solid dispersion & $70.6 \pm 16.3^{b, c)}$ & $1256.7 \pm 403.8^{b, c)}$ & $7.6 \pm 4.0$ \\
Drug: Eudragit E physical mixture & $28.4 \pm 12.6$ & $484.5 \pm 177.9$ & $5.8 \pm 1.6$ \\
Micronized raw drug & $14.8 \pm 4.4$ & $292.1 \pm 70.4$ & $10.0 \pm 2.7$ \\
\hline
\end{tabular}

a) Mean \pm S.D. $n=5$. b) Significant at $p<0.05$ vs. micronized raw material. $c$ ) Significant at $p<0.05$ vs. Eudragit E physical mixture. $d$ ) Significant at $p<0.05$ s. HPC solid dispersion. e) Significant at $p<0.05$ vs. HPMC solid dispersion.

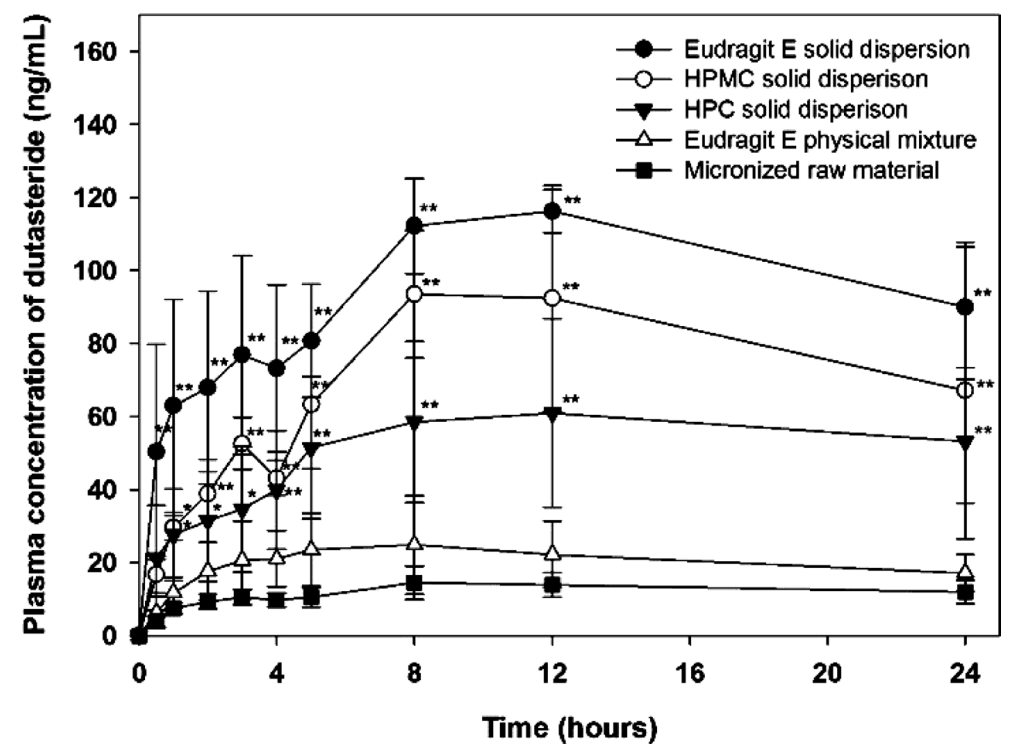

Fig. 3. Plasma Concentration-Time Profile of Dutasteride in Rats after Oral Administration of Micronized Raw Material, Physical Mixture, and Solid Dispersions at a Dose Equivalent to $2 \mathrm{mg}$ of Dutasteride/kg of Body Weight (Data Represented Mean \pm S.D., $n=5$ )

$* p<0.05, * * p<0.01$, significantly different compared to micronized raw material.

a supersaturated solution with an apparent solubility above the equilibrium solubility and thus has an increased driving force for transport across biological membranes, such as the epithelial membrane within the GI tract, thereby increasing the bioavailability of dutasteride. ${ }^{15,16)}$ These results suggest that preparation of Eudragit E solid dispersion using a spraydrying process may be a promising approach for improving the bioavailability of dutasteride. However, future research should fully elucidate the physicochemical properties and stability of Eudragit E solid dispersions to help develop solid dosage forms.

In conclusion, the relationship between supersaturation behavior, measured during dissolution, and in vivo absorption enhancement was investigated for various solid dispersions of dutasteride, prepared using a spray-drying process. A solid dispersion with Eudragit E, an acid-soluble polymer, displayed a high maximum supersaturation with an extended supersaturation, compared with water-soluble polymers such as HPMC, HPC, PVP, and HP- $\beta$-CD. Additionally, the absorption of dutasteride after oral administration of the Eudragit $\mathrm{E}$ solid dispersion to rats was markedly increased, due to the enhanced supersaturation and dissolution properties. The results from this preliminary study suggest that the preparation of a Eudragit E solid dispersion could be a promising approach to improve the supersaturation, dissolution, and absorption properties of dutasteride. 
Acknowledgments This work was supported by the 2012 Inje University Research Grant.

\section{References}

1) Olsson Gisleskog P., Hermann D., Hammarlund-Udenaes M., Karlsson M. O., Eur. J. Pharm. Sci., 8, 291-299 (1999).

2) Olsen E. A., Hordinsky M., Whiting D., Stough D., Hobbs S., Ellis M. L., Wilson T., Rittmaster R. S., Dutasteride Alopecia Research Team, J. Am. Acad. Dermatol., 55, 1014-1023 (2006).

3) U.S. Food Drug Administration, "Application Number: 21-319.": 〈http://www.accessdata.fda.gov/drugsatfda_docs/nda/2001/ 21319_Duagen_biopharmr_P1.pdf), cited 27 Jun, 2012.

4) Ofner C. M. 3rd, Zhang Y. E., Jobeck V. C., Bowman B. J., J. Pharm. Sci., 90, 79-88 (2001).

5) Kim C.-K., Park J.-S., Am. J. Drug Deliv., 2, 113-130 (2004).

6) Kim K. T., Lee J. Y., Lee M. Y., Song C. K., Choi J., Kim D.-D., J. Pharm. Invest., 41, 125-142 (2011).

7) Park C.-W., Tung N.-T., Son D.-D., Kim J.-Y., Rhee Y.-S., Kang S.-Y., Park S.-A., Hwang K.-M., Oh T.-O., Ha J.-M., Chi S.-C., Park
E.-S., J. Pharm. Invest., 42, 121-126 (2012).

8) Vasconcelos T., Sarmento B., Costa P., Drug Discov. Today, 12, 1068-1075 (2007).

9) Ramakrishna N. V. S., Vishwottam K. N., Puran S., Koteshwara M., Manoj S., Santosh M., J. Chromatogr. B Analyt. Technol. Biomed. Life Sci., 809, 117-124 (2004).

10) Kojima T., Higashi K., Suzuki T., Tomono K., Moribe K., Yamamoto K., Pharm. Res., 29, 2777-2791 (2012).

11) Overhoff K. A., McConville J. T., Yang W., Johnston K. P., Peters J. I., Williams R. O. 3rd, Pharm. Res., 25, 167-175 (2008).

12) Holmes S. A., Thomas T. D., J. Am. Chem. Soc., 97, 2337-2341 (1975).

13) Kim M.-S., Jin S. J., Kim J.-S., Park H. J., Song H. S., Neubert R. H., Hwang S.-J., Eur. J. Pharm. Biopharm., 69, 454-465 (2008).

14) Kim M. S., Kim J. S., Park H. J., Cho W. K., Cha K. H., Hwang S. J., Int. J. Nanomedicine, 6, 2997-3009 (2011).

15) Gao P., Guyton M. E., Huang T., Bauer J. M., Stefanski K. J., Lu Q., Drug Dev. Ind. Pharm., 30, 221-229 (2004).

16) Janssens S., De Zeure A., Paudel A., Van Humbeeck J., Rombaut P., Van den Mooter G., Pharm. Res., 27, 775-785 (2010). 